\title{
Ernährungsberatung im Spannungsfeld zwischen Klinik und ambulanter Versorgung
}

\section{Nutritional Counselling at the Edge Between Clinic and Outpatient Care}

Autor

Heike Weiss

Institut

Klinik für Allgemein- und Viszeralchirurgie, Klinikum

Magdeburg gGmbH

Schlüsselwörter

Krebs, Ernährung, Mangelernährung, Patienten,

Entlassmanagement

Keywords

cancer, nutrition, malnutrition, patient, arrangement of discharge

Bibliografie

DOI https://doi.org/10.1055/a-0894-1224

Aktuel Ernahrungsmed 2019; 44: 261-268

(c) Georg Thieme Verlag KG Stuttgart · New York ISSN 0341-0501

Korrespondenzadresse

Dr. rer. nat. Heike Weiss, Dipl. oec. troph., Klinikum

Magdeburg gGmbH, Klinik für Allgemein- und

Viszeralchirurgie, Birkenallee 34, 39130 Magdeburg

heike.weiss@klinikum-magdeburg.de

\section{ZUSAMMENFASSUNG}

Dass mit der Diagnose Krebs gleichzeitig eine erhöhte Gefahr für die Entstehung oder auch Verschlimmerung einer Mangelernährung einhergeht, ist eine seit fast 40 Jahren bekannte Tatsache. Umso wichtiger ist es, dass mehr dafür getan wird, um die kontinuierliche Versorgung dieser Patienten mit ernährungstherapeutischen Maßnahmen zu verbessern.

Es zeigt sich, dass im stationären Bereich mittlerweile Strukturen bestehen und Spezialisten verschiedenster Professionen daran arbeiten, die Ernährungssituation der Patienten zu verbessern. Einen massiven Einschnitt erfahren viele Patienten jedoch unmittelbar nach ihrer Entlassung. Ernährungstherapeutische Maßnahmen, die in der Klinik initiiert wurden, enden oft mit dem Tag der Entlassung. Die Ursachen hierfür sind vielfältig. Fehlende Strukturen im ambulanten Bereich, unzureichende Kommunikation zwischen Klinik und übernehmendem Haus- oder Facharzt oder eine ungenügende Aufklärung der Patienten über Sinn, Funktion und Ablauf der Ernährungstherapie verhin- dern deren kontinuierliche Fortsetzung nach der Entlassung.

Ein erster Schritt, um diesen Übergang zu verbessern, vollzog sich 2017 mit der gesetzlichen Verankerung des Entlassmanagements. Nun gilt es in diesem Sinne auch die Bedingungen für das Entlassmanagement der Patienten mit supportiver Ernährungstherapie so zu formulieren und zu strukturieren, dass keine Brüche im Behandlungsverlauf durch Entlassungen entstehen können. Dies führt letzten Endes nicht nur zu einer höheren Lebensqualität und Zufriedenheit bei den Patienten, sondern auch durch eine Verbesserung der Ernährungssituation zu weniger und kürzeren Klinikaufenthalten, die sich dann auch gesamtwirtschaftlich positiv auswirken.

\section{ABSTRACT}

The fact that the diagnosis of cancer is accompanied by an increased risk of developing or worsening malnutrition is unfortunately an apparently accepted fact. Therefore, it is important that everything is done to improve and continue nutritional care of these patients.

It has become apparent that structures were established in the inpatient area where a multiprofessional team is working to improve the nutritional situation of the patients. However, the patients then experience a massive incision regarding their nutritional care when discharged from the hospital. Nutritional therapy initiated in the clinic often ends abruptly at the day of discharge. The causes for this are manifold. Lack of structures in the outpatient area, inadequate communication between the clinic and the general practitioner or the inadequate education of patients about the meaning, function and course of nutritional therapy prevent their continuous continuation after discharge. A first step to close this gap took place in 2017 with the legal anchoring of the dismissal management. In this sense, it is now also necessary to formulate and structure the conditions for discharge management of patients with supportive nutritional therapy in such a way that no breaks in the course of treatment can result from layoffs.

In the end, this not only leads to a higher quality of life and satisfaction among the patients, but also through an improvement in the nutritional situation to fewer and shorter hospital stays, which then also have a positive macroeconomic effect. 


\section{Einleitung}

Die Entwicklung einer Mangelernährung bei Tumorpatienten ist relativ häufig, da diese nicht nur erkrankungs-, sondern auch therapieabhängig getriggert wird [1]. Die Prävalenz einer Mangelernährung von Tumorpatienten, die in einer Klinik aufgenommen werden, liegt bei ca. $50 \%$ [2].

In der Regel verändert ein Krankenhausaufenthalt die Ernährungssituation eines mangelernährten Patienten nicht [3, 4]. $63 \%$ der Patienten, die mindestens 7 Tage in der Klinik waren, verlassen diese im gleichen Ernährungsstatus, bei $20 \%$ der Patienten hat sich der Ernährungszustand aber verschlechtert [5].

Bereits Anfang der 80er-Jahre zeigte Dewys [6], dass die Prävalenz einer Mangelernährung bei Aufnahme und während eines Krankenhausaufenthalts sehr hoch ist. Auch nach über 40 Jahren bleibt dieses Problem bestehen [7]. Die Prävalenz liegt weiterhin bei ca. $50 \%$. Trotz etablierter Ernährungsscreenings und strukturierter Ernährungsinterventionsprogramme hat sich die Situation kaum gebessert.

Betrachtet man die orale Nahrungsaufnahme von Klinikpatienten, so verwundert es nicht, dass mit einer mittleren Energieaufnahme von ca. $750 \mathrm{kcal}$ (Kilokalorien) pro Tag (759 $\pm 546,8 \mathrm{kcal}$ pro Tag) keine Verbesserung der ggf. bestehenden Mangelernährungssituation erfolgen kann [7].

Betreibt man Ursachenforschung bei stationären Patienten, so geben diese vor allem Probleme beim Essen und Appetitmangel als Grund für ihren schlechten Ernährungszustand an [8-11]. Da diese Ursachen in der Regel nicht spontan bei der Entlassung verschwinden, besteht das Risiko für ein Fortbestehen oder sogar Eskalation der Mangelernährung natürlich weiterhin.

Dies zeigt, dass sämtliche Interventionen, die auf eine Verbesserung der Ernährungssituation abzielen, zeitlich nicht nur während des Klinikaufenthalts stattfinden sollten, sondern auch vor (Stichwort Prähabilitation) und nach einem Klinikaufenthalt [12].

Betrachtet man nun die ernährungstherapeutische Versorgung im Krankenhaus, zeigt sich folgendes Bild. Während im Krankenhaus noch ca. $50 \%$ der Patienten Ernährungsinformationen oder eine Beratung erhielten [12-14], haben nur noch $7 \%$ der Patienten im Anschluss an die Entlassung aus dem Krankenhaus Kontakt mit einer Ernährungsfachkraft, obwohl der Ernährungszustand sich teilweise seit der Entlassung weiter verschlechtert hatte [12].

Eine weitere Herausforderung scheint auch die Fortsetzung einer Ernährungstherapie (z.B. orale Nahrungssupplemente, Trinknahrung) in der Häuslichkeit. Die Studie von Ginzburg [15] zeigt, dass nur $21 \%$ der Patienten mit einer expliziten Empfehlung für ONS (orale Nahrungssupplemente) im Entlassungsbrief, diese auch vom Hausarzt rezeptiert bekamen. Obwohl klinische Studien zeigen, dass Ernährungsberatung und/oder die Gabe von ONS bei Mangelernährung positive Effekte bei den Patienten zeigt [16, 17], bricht eine orale Ernährungstherapie in den meisten Fällen bei der Entlassung aus der Klinik ab. Betroffen hiervon sind vor allem Patienten, die nicht dem klassischen Bild eines mangelernährten Patienten mit einem niedrigen BMI (Body-Mass-Index) entsprechen [15].

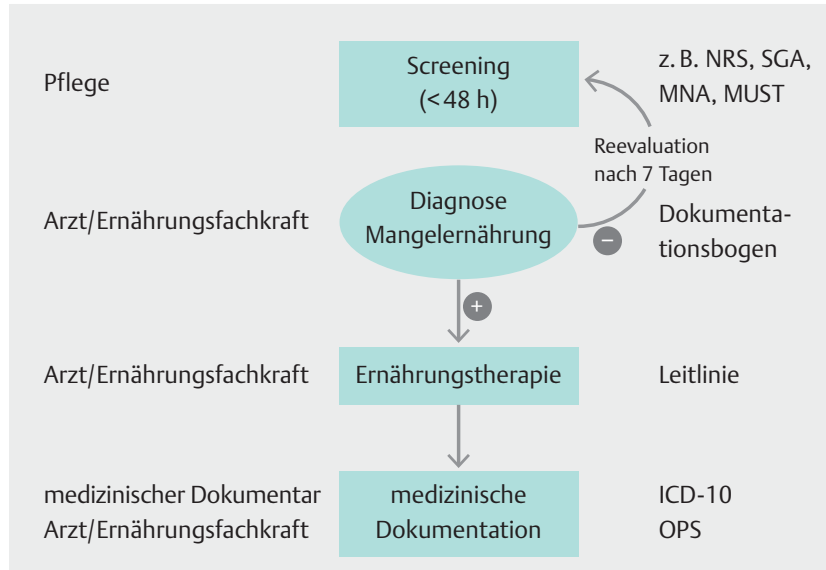

Abb. 1 Prozessschema Mangelernährung in der Klinik (nach [20]).

Auch der Zugang zu weiterer Ernährungsberatung im ambulanten Bereich wird den Patienten nur schwer ermöglicht. Allein die Ausbildung der DiätassistentInnen oder das Studium der Ökotrophologie qualifiziert diese als Ernährungsfachkraft, die, idealerweise in enger Zusammenarbeit mit dem Hausarzt, die Ernährungstherapie der Patienten von Anfang an begleiten [18].

\section{Ernährungstherapie/Versorgung im Krankenhaus am Beispiel einer interdis- ziplinären viszeralchirurgisch/onkologi- schen Station}

Prekärerweise zeigt sich, dass sich die Gefahr für eine Mangelernährung bei stationären Patienten durch eine oftmals unzureichende Ernährung und eine fehlende Ernährungsintervention weiter verschärft. Durch ein frühzeitiges Mangelernährungsscreening können gefährdete Patienten bei Aufnahme erfasst werden und einem auf sie zugeschnittenen Ernährungsmanagement zugeführt werden. Gleichzeitig ermöglicht das routinemäßige Screening mit validierten Screeningtools eine Codierung und Abbildung der Mangelernährung im DRG-System (diagnosebezogene Fallgruppen) [7]. Um eine gleichbleibende Qualität der ernährungstherapeutischen Versorgung der Patienten zu gewährleisten, ist es unerlässlich, den gesamten Vorgang vom Screening über das Assessment hin zur Ernährungsintervention, des Monitorings und der abschließenden Dokumentation verlässlich zu strukturieren [19]. Ein Prozessschema zur Therapiestruktur bei Mangelernährung findet sich auch in - Abb. 1 [20].

Der Ablauf eines solchen Prozesses soll hier exemplarisch, wie er am Klinikum Magdeburg abläuft, aufgezeigt werden. Als Beispielstation fungiert hier eine interdisziplinäre viszeralchirurgisch-onkologische Station. 
Tab. 1 Mögliche Erhebungsparameter im Rahmen des Ernährungsassessments.

\begin{tabular}{|c|c|}
\hline \multicolumn{2}{|l|}{ objektive Methoden } \\
\hline $\begin{array}{l}\text { anthropometrische } \\
\text { Verfahren }\end{array}$ & $\begin{array}{l}\text { - Gewicht, Größe, BMI, Gewichtsverlauf } \\
\text { - Waden-, Oberarmumfang, Taillenumfang, } \\
\text { Waist-to-Hip-Ratio } \\
\text { - Hautfaltendicke (Oberarm) }\end{array}$ \\
\hline $\begin{array}{l}\text { Bestimmung der } \\
\text { Körperzusammen- } \\
\text { setzung }\end{array}$ & $\begin{array}{l}\text { - BIA (bioelektrische Impedanzanalyse) } \\
\text { - sonografische Messung des Depotfetts }\end{array}$ \\
\hline Labordiagnostik & $\begin{array}{l}\text { - Blutuntersuchung (Protein, Eisen, Vitami- } \\
\text { ne) } \\
\text { - Blutbild } \\
\text { - Harnuntersuchung (Protein, Glukose, } \\
\text { Ketonkörper) }\end{array}$ \\
\hline $\begin{array}{l}\text { funktionelle Unter- } \\
\text { suchung }\end{array}$ & $\begin{array}{l}\text { - Muskelkraft (Hand-, Zugdynamometer) } \\
\text { - Belastungstests }\end{array}$ \\
\hline \multicolumn{2}{|l|}{ subjektive Methoden } \\
\hline $\begin{array}{l}\text { Ernährungsanam- } \\
\text { nese, -assessment }\end{array}$ & $\begin{array}{l}\text { - Appetit, Trinkmenge, Lebensmittelpräfe- } \\
\text { renzen } \\
\text { - Abwechslung, Portionen, Probleme bei } \\
\text { Nahrungsaufnahme (Dysphagie) }\end{array}$ \\
\hline $\begin{array}{l}\text { Ernährungsproto- } \\
\text { kolle, -dokumen- } \\
\text { tation }\end{array}$ & $\begin{array}{l}\text { - Erfassung von Ess- und Trinkmengen, } \\
\text { Unverträglichkeiten, akuten Problemen } \\
\text { (Durchfall, Verstopfung) } \\
\text { - Beobachtung Ernährungsverhalten am } \\
\text { Tisch (Vorlieben, Abneigungen, Würzen) }\end{array}$ \\
\hline $\begin{array}{l}\text { klinische Anamnese } \\
\text { und ärztliche Unter- } \\
\text { suchung }\end{array}$ & $\begin{array}{l}\text { - Erkrankungen und Medikation } \\
\text { - } \text { psychomentale/soziale Situation } \\
\text { - } \text { allgemeines Erscheinungsbild, Haltung } \\
\text { - Haut (Turgor, Blässe, Ekzeme), Schleim- } \\
\text { - haut, Wundheilung } \\
\text { - } \text { Haarausfall, Nägel } \\
\text { - neurologische Untersuchung }\end{array}$ \\
\hline
\end{tabular}

\section{Erfassung von Mangelernährung anhand von Screenings}

Für Krankenhauspatienten ist das auch im Klinikum Magdeburg verwendete Nutritional Risk Screening [19] empfohlen. Die Durchführung erfolgt in der Regel innerhalb von 48 Stunden nach Aufnahme der Patienten durch das Pflegepersonal oder das Ernährungsfachpersonal. Besteht ein positives Screeningergebnis, wird durch die ernährungstherapeutische Fachkraft ein Ernährungsassessment durchgeführt ( $\triangleright$ Tab. 1 ).

\section{Durchführung eines Ernährungsassessments}

Das Ernährungsassessment dient zur detaillierten Erhebung des Ernährungszustands und betreibt Ursachenforschung bezüglich der Mangelernährung. Es dient als Basis für die daraus folgenden Empfehlungen zur Ernährungstherapie [21, 22]. Die erhobenen Parameter gewährleisten außerdem eine adäquate Abbildung des Ernährungszustands des Patienten im DRG-System. Die erhobenen Parameter finden sich in > Tab. 1 [21 - 25].

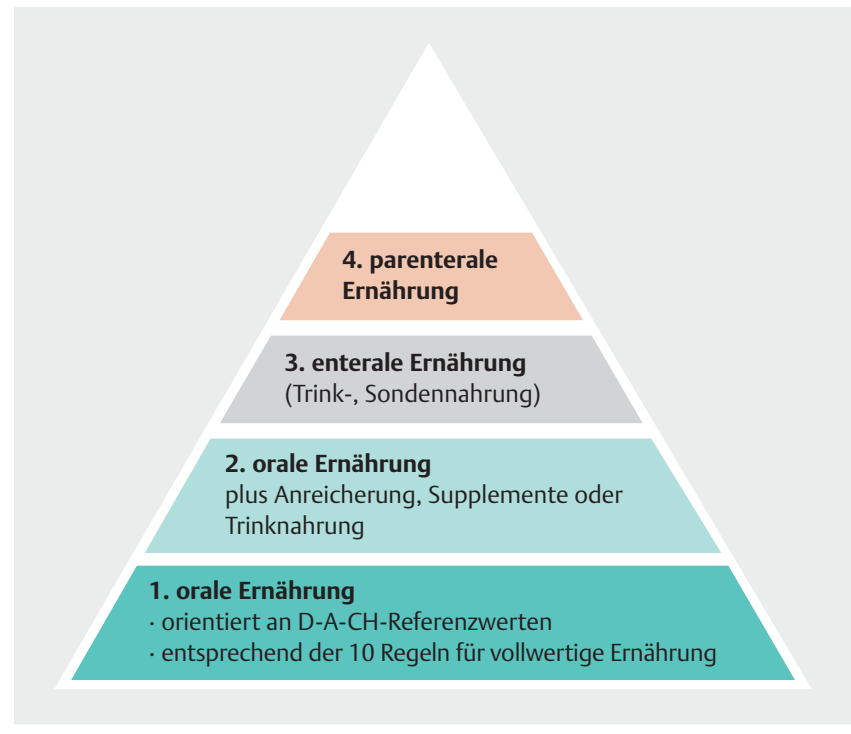

Abb. 2 Stufenschema Ernährungstherapie, Mischformen der verschiedenen Stufen sind ausdrücklich erwünscht. Das Potenzial der oralen Ernährung sollte immer so weit wie möglich ausgeschöpft werden. Der Ernährungszugang sollte sich immer an der natürlichsten Form orientieren (oral vor enteral vor parenteral) [22, 25].

\section{Ernährungsberatung und Therapie}

Einer Tatsache sollten sich alle, die in den Prozess der Ernährungstherapie involviert sind, bewusst sein. Es ist immer effizienter, ein frühes Stadium der Mangelernährung zu behandeln, als gegen eine massive Form der Mangelernährung (z. B. Kachexie) therapieren zu müssen [26]. Die Erfahrung zeigt, dass vor allem Patienten, die zwar mit dem BMI im Normalbereich sind, aber bereits mehr als $10 \%$ Gewichtsverlust innerhalb der letzten 3-6 Monate erlitten haben, besondere Beachtung erfahren sollten. Per definitionem [22] sind auch diese Patienten beim Vorliegen einer malignen Erkrankung mangelernährt und bedürfen einer Ernährungsintervention. Oftmals reicht hier bereits eine ausführliche Ernährungsberatung mit Hinweisen für eine energie- und eiweißreiche Ernährung, um dem weiteren Gewichtsverlust Einhalt zu gebieten. Auch das Aushändigen von Kontaktdaten, um dem Patienten einen Ansprechpartner beim Persistieren oder Verschlimmern der Ernährungssituation zu gewährleisten, kann hilfreich sein.

Die Erfüllung der ernährungstherapeutischen Bedürfnisse der Tumorpatienten sollte idealerweise multidisziplinär unter Beachtung des etablierten Stufenschemas ( Abb. 2) [27] erfolgen. Im Mittelpunkt steht zunächst die orale Ernährung und deren Anreicherung. Erst schrittweise finden dann orale, enterale und parenterale Nährstoffsubstrate Verwendung.

\section{Themen und Ablauf Ernährungsberatung im Krankenhaus}

Ein Großteil der Inhalte für die Ernährungsberatung auf der viszeralchirurgisch-onkologischen Station hat zunächst nur indirekt mit dem Thema Mangelernährung zu tun. Die meisten Themen fokussieren sich auf die Veränderungen der Ernährungsge- 
wohnheiten durch die eingesetzten OP-Verfahren zur Behandlung der verschiedenen Tumorentitäten des Gl-Traktes (gastrointestinale Tumore).

1. Operationen im oberen GI-Bereich (z. B. Ösophagusresektion und Magenhochzug, Gastrektomie partiell und total)

2. Pankreasresektionen (Kopf, Korpus, Schwanz)

3. Darmoperationen (Dünndarmresektionen, Hemikolektomie, Anlage Anus praeter in Dünn- oder Dickdarm)

Im Idealfall verteilt sich der Beratungsablauf auf mehrere Konsultationen. Dies hat sich im Laufe der Zeit aus mehreren Gründen als sinnvoll erwiesen, auf die im Folgenden eingegangen wird.

Die erste Konsultation erfolgt, wenn möglich, noch vor der geplanten OP. Hier werden dem Patienten erste Informationen zu den zu erwartenden Veränderungen bei Nahrungsaufnahme und Essverhalten nach der OP vorgestellt. Auch Informationen zum Kostaufbau nach der OP werden dem Patienten ausgehändigt und mit ihm besprochen.

Die zweite Konsultation erfolgt in der Regel zum Zeitpunkt der Kostaufbaustufe Weißbrot/Brei. Vorher sind die Patienten in der Regel noch zu sehr von den Nachwirkungen der OP (Schmerzen, Atonie, Schwäche u. Ä.) beeinträchtigt, sodass noch keine adäquate Aufnahme der Informationen stattfinden kann. Es werden nun Themen wie Mahlzeitengröße, geeignete Lebensmittel, Mahlzeitenfrequenz u. Ä. besprochen. Es erfolgt in der Regel die Aushändigung von Infomaterial mit dem alles Besprochene zusammengefasst ist, die Dauer der Konsultation ist sehr individuell und abhängig davon, wie der Zustand des Patienten ist. Meist sind nach 15-20 Minuten die aktuell wichtigsten Punkte besprochen. Die Infomaterialien sind bewusst sehr kompakt gestaltet. Eigene Erfahrungen haben gezeigt, dass zu umfangreiche und detaillierte Informationen gerade in dieser recht frühen Phase eher zur Verwirrung bei den Patienten geführt haben.

Zum nächsten Termin werden dann konkrete Fragen des Patienten beantwortet. Meist entstehen diese durch Probleme oder Beobachtungen beim Essen aber auch beim Studieren der Infomaterialien. Es wird dann gemeinsam mit dem Patienten überprüft, ob die benötigte Energiemenge durch orale Kost auch in der Häuslichkeit erreicht werden kann. Ist dies nicht der Fall, wird überprüft, ob der Energiebedarf durch gezieltes Anreichern der Nahrung (etwa mit dem Ersetzen energiearmer Lebensmittel mit einem energiereicheren Pendant, z. B. fettarme Milch mit Vollmilch oder Sahne) erreicht werden kann, ggf. erfolgt die Organisation der Versorgung mit ONS/Trinknahrung oder auch enteraler/parenteraler Ernährung. Dieser Termin ist in der Regel der zeitintensivste Beratungszeitpunkt. Wir rechnen hier, gerade wenn zusätzlich Angehörige dazu kommen und eine Organisation der häuslichen Versorgung notwendig ist, mit ca. 60-90 Minuten Beratungs- und Bearbeitungszeit.

Die Anwesenheit von Familienmitgliedern, z.B. dem Ehepartner oder Kindern, ist zu jedem Zeitpunkt der Ernährungsberatung möglich und erwünscht. Oft macht dies ganz besonders Sinn, wenn der Patient nicht derjenige im Haushalt ist, der das Essen zubereitet oder einkauft.
Kurz vor Entlassung findet dann noch eine Abschlussvisite statt. Die Patienten erhalten am Klinikum Magdeburg zusätzlich die Telefonnummer der Ernährungstherapie, die sie bei Fragen telefonisch weiterhin berät.

\section{Monitoring der Ernährungsintervention}

Die Effektivität der Ernährungsintervention wird über Gewichtskontrollen, Messung der Körperzusammensetzung, Ernährungsprotokolle, ggf. Laborkontrollen und das Beobachten von eventuellen Nebenwirkungen dokumentiert. Sinnvolle, zu erhebende Parameter sind:

Gewicht, BMI, Tellerprotokoll, Körperzusammensetzung (BIA-Messung [bioelektrische Impedanzanalyse: Körperzellmasse (BCM), Verhältnis ECM (extrazelluläre Masse)/BCM; Phasenwinkel]), Labor (Albumin, Protein).

Eine Anpassung und Modifikation der Ernährungstherapie aufgrund veränderter Parameter sollte jederzeit möglich sein. Im Durchschnitt führt eine Ernährungstherapie bei mangelernährten Patienten zu einer Verkürzung des Krankenhausaufenthalts von ca. 2,5 Tagen. Dies reduziert natürlich auch die Kosten. $[28-30]$.

\section{Situationen in der Klinik, die Mangel- ernährung verschärfen können und mögliche Lösungsansätze Diagnostik}

Vor allem Patienten mit Kopf- Hals- oder GI-Tumoren haben ein überdurchschnittlich hohes Risiko für die Entstehung einer Mangelernährung [31].

Für einige präoperative Standarduntersuchungen ist Nüchternheit und eine vorausgehende Darmreinigung unerlässlich. Da die Untersuchungen teilweise aus organisatorischen Gründen an verschiedenen Tagen stattfinden, sind oftmals mehrere Tage Nüchternheit für diese Patienten trotz bereits vor Aufnahme bestehender verschärfter Ernährungssituation keine Seltenheit. Folgende Möglichkeiten können hier ergriffen werden, um die Energielücke zu minimieren:

- parenterale Ernährung, ggf. mit peripheren Rezepturen

- energiereiche Trinkkonzentrate ohne Auswirkungen auf den Darm (z. B. fettfreie Trinknahrung)

- gute Koordination der Untersuchungen, um den Nüchternzeitraum zu minimieren

\section{Perioperative Ernährung}

Das Fast Track- oder ERAS (Enhanced Recovery after Surgery) -Konzept [32] führt das Prinzip der Vermeidung der im Klinikalltag häufig zu großzügig angeordneten Nahrungskarenz weiter. Gerade im Bereich der Eingriffe am Darm verhindern postoperative Symptome wie Übelkeit, Völlegefühl, Erbrechen, Darmatonie, Schmerzen oder Fatigue eine frühe Rekonvaleszenz. Es hat sich gezeigt, dass diese Komplikationen unter anderem durch die bis Anfang der 2000er-Jahre traditionelle orale Nahrungskarenz nach solchen Eingriffen noch verstärkt wurden [33]. Das oberste Prinzip zur frühen Rekonvaleszenz nach abdominellen Eingriffen ist die Verminderung von perioperativem 
Stress [34]. Zusätzlich empfehlen die Leitlinien der europäischen Gesellschaft für Klinische Ernährung und Metabolismus [34, 35]:

1. die Vermeidung von längeren präoperativen Nüchternheitsperioden

2. eine postoperativ frühestmögliche orale Kostzufuhr

3. die Einbeziehung der Ernährung in das perioperative Therapiekonzept

\section{Entlassmanagement}

Wie eingangs erwähnt, zeigt sich gerade der Übergang von der stationären Krankenhausversorgung in eine weitergehende ambulante Versorgung auch im ernährungstherapeutischen Bereich als sehr kritisch [15]. Seit dem 1.10.2017 ist das Entlassmanagement für deutsche Krankenhäuser verbindlich [36] und umschließt auch eine Versorgung der Patienten mit weiterführender häuslicher Ernährungstherapie. Hier liegt es sicherlich auch in den Händen der Klinik, diesen Übergang durch möglichst konkrete Empfehlungen für die Ernährungstherapie zu unterstützen. Je konkreter die Empfehlungen zur Ernährungstherapie der Patienten im Entlassungsbrief gefasst sind, desto besser können die übernehmenden Haus- und Fachärzte diese umsetzen. Auch eine direkte Anbindung in eine spezialisierte ernährungstherapeutische Praxis wäre sinnvoll.

Eine zusätzliche Unterstützung ist hier auch das Vermerken von ernährungsrelevanten Daten der Patienten. Als minimal sinnvoll wären das:

- Größe, Gewicht, BMI, Gewichtsverlust in den letzten

3-6 Monaten

- Portionsgröße

- Informationen über Lebensmittelunverträglichkeiten

- individuelle Ernährungsempfehlungen

Als zusätzliche Daten sinnvoll, wenn erhoben, sind die Ergebnisse von Körperzusammensetzungsmessungen (BIA-Messung), Laborparameter wie Albumin, Protein und auch der relevante Mangelernährungscode (z.B. E44.0).

\section{Ernährungstherapie/Versorgung im Ambulanten Bereich am Beispiel eines onkologischen MVZ}

Im Januar 2015 entschied der gemeinsame Bundesausschuss, dass es keinen Beleg für den Nutzen einer alleinigen Ernährungsberatung hinsichtlich patientenrelevanter Endpunkte bei unterschiedlichen ernährungsabhängigen Erkrankungen gibt. Hierzu zählen auch die onkologischen Erkrankungen. Somit besteht formal keine medizinische Notwendigkeit für eine ambulante Ernährungsberatung und sie wurde nicht als Heilmittel in den Leistungskatalog der gesetzlichen Krankenkassen aufgenommen.

Der existierende Zuschuss der Krankenkassen für eine Ernährungstherapie deckt in der Regel nur einen Teil der entstehenden Kosten und ist auch regional und von Krankenkasse zu Krankenkasse unterschiedlich. Insofern ist die Höhe der Kosten-

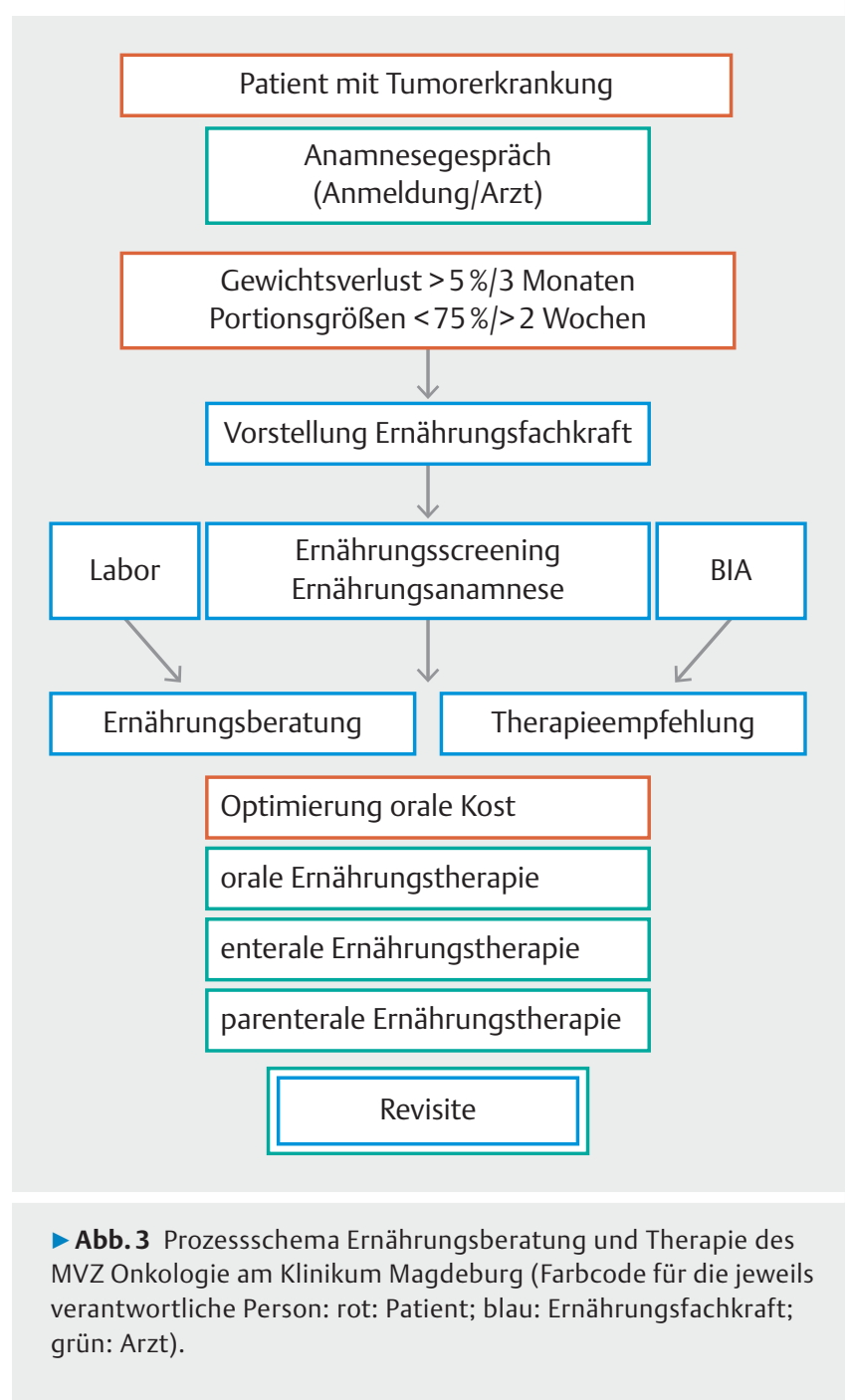

erstattung von Patient zu Patient sehr unterschiedlich und sollte im Vorfeld jeweils individuell abgeklärt werden [37].

Während die multiprofessionelle Betreuung von Tumorpatienten durch onkologische Zentren und die Zertifizierung dieser zu einer Selbstverständlichkeit geworden ist, zeigt die Studie von Erickson [18], dass nur 70\% dieser Zentren eine Ernährungsfachkraft besitzen. Ein damit einhergehendes strukturiertes Programm zur Ernährungsberatung liegt dann nur noch in ca. $50 \%$ der Zentren vor. Somit bleiben viele Patienten beim Thema Ernährung sich selbst überlassen. Noch problematischer werden die Möglichkeiten auf eine ernährungstherapeutische Weiterbehandlung der Patienten nach der Entlassung aus der Klinik. Nur bei einem Viertel der Zentren ist diese auch für ambulante Patienten gewährleistet. Ungeklärt ist dabei in der Regel die Finanzierung. Eine ernährungstherapeutische Versorgung im ambulanten Bereich, gerade auch im Rahmen einer Beratung ist danach noch seltener, da hier, wie zuvor geschildert, die Finanzierung durch die Krankenkassen in der Regel nicht gesondert gedeckt wird, sondern in der onkologischen EBM-Ziffer enthalten ist. Es bleibt also dem Engagement der einzelnen onkologischen Facharztpraxen überlassen, ob und in 
welcher Form eine ernährungstherapeutische Versorgung der Patienten fortgeführt wird.

\section{Erfassung der Mangelernährung/Screening}

In der onkologischen Facharztpraxis am Klinikum Magdeburg entfällt ein isoliertes Screening auf Mangelernährung, dieses wird im Rahmen des Anamnesegesprächs vom Arzt durchgeführt und dieser entscheidet, ob eine Vorstellung bei der Ernährungsfachkraft erfolgt oder nicht. Als Entscheidungskriterien fungieren hier zusätzlich zum BMI der Gewichtsverlust über einen definierten Zeitraum ( $>5 \%$ innerhalb von 3 Monaten) und die Portionsgröße ( $<75 \%$ für mehr als 2 Wochen) ( $\triangleright$ Abb. 3). Auch hier wird bewusst nicht der BMI alleine als Kriterium angesetzt, da dieser nur eine eingeschränkte Aussagekraft besitzt [38]. Zusätzlich spielt die langjährige ernährungsmedizinische Erfahrung des Onkologen eine wichtige Rolle.

\section{Durchführung des Ernährungsassessments}

Der Ablauf von Ernährungsscreening und -anamnese in der Praxis unterscheidet sich zunächst nicht von dem in der Klinik. Im Unterschied zu den Krankenhauspatienten wird hier ein 24-hRecall zur Überprüfung der aufgenommenen Nahrungsmenge durchgeführt. Im Krankenhaus sind solche Recalls oft schwierig, da sich viele Patienten nicht mehr an die Mahlzeiten vor Aufnahme erinnern.

\section{Themen der Ernährungsberatung}

In der Regel unterscheiden sich die Themen der Ernährungsberatung für onkologische Patienten im ambulanten Bereich deutlich von denen im stationären Bereich. Während im Krankenhaus Themen wie Kostaufbau, Ernährung nach Eingriffen am GI-Trakt, verträgliche Lebensmittel, Mahlzeitenfrequenz, Einnahme von Enzympräparaten und Mahlzeitengröße im Vordergrund stehen, interessieren sich Patienten einer onkologischen Facharztpraxis oft für Themen wie gesunde Ernährung, Nahrungsergänzungsmittel und Krebsdiäten [13, 18, 39, 40]. Ebenfalls im Fokus des Interesses der Patienten stehen der Umgang mit Nebenwirkungen der Chemotherapie wie Appetitverlust, Übelkeit und Erbrechen. Auch die Nachwirkungen eines onkologisch-chirurgischen Eingriffs am GI-Trakt (z. B. Gastrektomie, Operationen am Darm) sind weiterhin Thema der Ernährungsberatung.

Ein weiteres Thema, welches weniger von den Patienten vorgegeben wird, aber aufgrund der hohen Prävalenz der Mangelernährung oft von Arzt oder Ernährungsfachkraft in einem zweiten Schritt thematisiert wird, ist die adäquate Energiezufuhr im häuslichen Umfeld, oftmals bereits begleitet von Gesprächen über die Möglichkeiten der künstlichen Ernährung (ONS, heimenteral oder heimparenteral). Der Grund hierfür ist, dass auch im ambulanten Bereich versucht werden sollte, eine Mangelernährung so früh wie möglich zu behandeln [41]. Die Erfahrung zeigt leider, dass viele Patienten zu lange zögern, einer frühzeitigen ambulanten Ernährungstherapie zuzustimmen. Damit entgeht dem Patienten leider oft die Chance, frühzeitig einer Verschlechterung des Ernährungsstatus entgegenzuwirken und dabei zunächst nur relativ geringe zusätzliche Einflüsse durch die Ernährungstherapie in Kauf nehmen zu müssen (z. B. Mengen an ONS oder künstlicher Ernährung, ggf. auch die Frequenz der künstlichen Ernährung, die dann zunächst nicht täglich erfolgen müsste).

\section{Situationen in der Arztpraxis, die Mangel- ernährung verschärfen können und mög- liche Lösungsansätze}

\section{Kontaktaufnahme}

Die personelle Abdeckung der Ernährungstherapie im ambulanten Bereich ist deutlich schlechter als in der Klinik. Aufgrund der fehlenden, vollständigen Finanzierung der Ernährungstherapie durch eine Ernährungsfachkraft ist die Abdeckung der Sprechzeiten im ambulanten Bereich lückenhaft. Gleichzeitig ist die Bereitschaft der ohnehin finanziell schwer belasteten Patienten zu einer weiteren, wenn auch nur teilweisen Übernahme der Beratungskosten sehr gering.

Eine Verbesserung könnte hier die konsequentere Nutzung von telefonischer Kontaktaufnahme und Beratung im Rahmen der poststationären Betreuung bringen. Eine Studie in Australien zeigte, dass die Patienten es durchaus schätzen, eine Ernährungsberatung, auch wenn sie telefonisch erfolgt, zu erhalten [42].

\section{Revisiten}

Die oben beschriebene Problematik setzt sich fort bei der unerlässlichen Wiedervorstellung der Patienten. Dies ist zum einen besonders kritisch zu sehen bei Patienten, die wegen eines positiven Mangelernährungsscreenings aufgefallen sind, eine Ernährungsberatung erhielten aber keine Ernährungssupplemente akzeptieren. Die eigentlich notwendige, engmaschige Überwachung ist hier kaum durchzuführen. Dasselbe gilt für Patienten, die eine künstliche Ernährung im ambulanten Bereich erhalten. Gerade zu Beginn der Versorgung ist eine engmaschige Kontrolle (alle 4 Wochen) empfohlen [41]. Die Überwachung erfolgt hier in der Regel über den niedergelassenen, rezeptierenden Haus- oder Facharzt. Am Klinikum Magdeburg meldet außerdem das betreuende Homecareunternehmen monatlich den Ernährungszustand des Patienten rück. Treten hier Auffälligkeiten oder Veränderungen auf (Patient nimmt weiter ab, kann besser/schlechter essen etc.), erfolgt die Rückmeldung auch außerhalb der Reihe.

\section{Resümee}

Mit der Ernährungstherapie gibt es im Rahmen einer multidisziplinären Behandlung von Krebserkrankungen eine wichtige Komponente, die zu allen Therapiezeitpunkten eine unterstützende Wirkung haben kann. Dieses Potenzial bleibt leider allzu oft ungenutzt. Verbessern würde sich diese Situation durch eine Optimierung der Zusammenarbeit von Onkologen und Ernährungsfachkräften, gerade auch mit Fokus auf das Leistungsangebot von onkologischen Schwerpunktpraxen. Auch die Stärkung der Kommunikation zwischen allen in die Versorgung von Krebspatienten involvierten Professionen könnte die Gesamtsituation der Betroffenen verbessern. Ebenso bedroht der Über- 
gang von stationärer in die ambulante Versorgung die konsequente Fortführung einer Ernährungstherapie. Die Stärkung dieser Schnittstellen durch strukturierte Programme und die Verbesserung der Kommunikation innerhalb dieser Bereiche könnte dazu führen, dass begonnene Ernährungstherapien auch in der Häuslichkeit fortgeführt werden. Dies könnte die bereits gesicherten Effekte der Ernährungstherapie wie geringere Mortalität und Morbidität, weniger Wiederaufnahmen und letztendlich auch Kostenersparnis noch weiter verstärken.

Erstveröffentlichung: Dieses Manuskript wurde modifiziert nach Auszügen aus dem Vortrag „Bedürfnisse von Tumorpatienten in der Ernährungstherapie - Was kommt in der Praxis an?" der anlässlich der 17. DGEM-Jahrestagung 2018 in Kassel gehalten wurde.

\section{Interessenkonflikt}

Die Autorin hat in den vergangenen 3 Jahren Vortragshonorare von den Firmen BBraun, Fresenius und Publicare erhalten.

\section{Literatur}

[1] Arends ], Bertz H, Bischoff SC et al. Klinische Ernährung in der Onkologie. Aktuel Ernahrungsmed 2015; 40: 301 - 329

[2] Pirlich M, Schutz T, Kemps M et al. Prevalence of malnutrition in hospitalized medical patients: impact of underlying disease. Dig dis 2003; $21: 245-251$

[3] Allard JP, Keller H, Jeejeebhoy KN et al. Malnutrition at Hospital Admission-Contributors and Effect on Length of Stay: A Prospective Cohort Study From the Canadian Malnutrition Task Force. Jpen 2016; 40: $487-497$

[4] Barker LA, Gout BS, Crowe TC. Hospital malnutrition: prevalence, identification and impact on patients and the healthcare system. Int J Environ Res Public Health 2011; 8: 514- 527

[5] Allard JP, Keller $\mathrm{H}$, Teterina $\mathrm{A}$ et al. Factors associated with nutritional decline in hospitalised medical and surgical patients admitted for $7 \mathrm{~d}$ or more: a prospective cohort study. Br J Nutr 2015; 114: 1612 - 1622

[6] Dewys WD, Begg C, Lavin PT et al. Prognostic effect of weight loss prior to chemotherapy in cancer patients. Eastern Cooperative Oncology Group. Am J Med 1980; 69: 491 - 497

[7] Konturek PC, Herrmann HJ, Schink K et al. Malnutrition in Hospitals: It Was, Is Now, and Must Not Remain a Problem! Med Sci Monit 2015; 21: $2969-2975$

[8] Kagansky N, Berner $\mathrm{Y}$, Koren-Morag N et al. Poor nutritional habits are predictors of poor outcome in very old hospitalized patients. Am J Clin Nutr 2005; 82: 784-791; quiz 913-914

[9] Keller H, Allard J, Vesnaver E et al. Barriers to food intake in acute care hospitals: a report of the Canadian Malnutrition Task Force. J Hum Nutr Diet 2015; 28: 546- 557

[10] Westergren A, Lindholm C, Axelsson C et al. Prevalence of eating difficulties and malnutrition among persons within hospital care and special accommodations. J Nutr Health Aging 2008; 12: 39-43

[11] Westergren A, Unosson M, Ohlsson O et al. Eating difficulties, assisted eating and nutritional status in elderly ( $>$ or $=65$ years) patients in hospital rehabilitation. Int J Nurs Stud 2002; 39: $341-351$

[12] Laur C, Curtis L, Dubin J et al. Nutrition Care after Discharge from Hospital: An Exploratory Analysis from the More-2-Eat Study. Healthcare (Basel, Switzerland) 2018; 6: doi:10.3390/healthcare6010009
[13] Maschke J, Kruk U, Kastrati K et al. Nutritional care of cancer patients: a survey on patients' needs and medical care in reality. Int J Clin Oncol 2017; 22: $200-206$

[14] Thompson J, Silliman K, Clifford DE. Impact of an early education multimedia intervention in managing nutrition-related chemotherapy side effects: a pilot study. SpringerPlus 2013; 2: 179

[15] Ginzburg Y, Shmilovitz I, Monastyrsky N et al. Barriers for nutritional care in the transition from hospital to the community among older patients. Clin Nutr ESPEN 2018; 25: 56-62

[16] Baldwin C, Weekes CE. Dietary advice with or without oral nutritional supplements for disease-related malnutrition in adults. Cochrane Database Syst Rev 2011: CD002008

[17] Stratton RJ, Elia M. Who benefits from nutritional support: what is the evidence? Eur J Gastroenterol Hepatol 2007; 19: 353-358

[18] Erickson N, Paradies K, Buchholz D et al. Nutrition care of cancer patients - A survey among physicians and nurses. Eur J Cancer Care 2018; 27: e12855

[19] Kondrup J, Johansen N, Plum LM et al. Incidence of nutritional risk and causes of inadequate nutritional care in hospitals. Clin Nutr 2002; 21 : $461-468$

[20] Ockenga J, Freudenreich M, Zakonsky R et al. Nutritional assessment and management in hospitalised patients: implication for DRG-based reimbursement and health care quality. Clin Nutr 2005; 24: 913-919

[21] Kondrup J, Allison SP, Elia M et al. ESPEN guidelines for nutrition screening 2002. Clin Nutr 2003; 22: 415-421

[22] Valentini L, Volkert D, Schütz T et al. Guideline of the German Society for Nutritional Medicine DGEM Terminology for Clinical Nutrition. Aktuel Ernahrungsmed 2013; 38: 97 -111

[23] Cederholm T, Bosaeus I, Barazzoni R et al. Diagnostic criteria for malnutrition - An ESPEN Consensus Statement. Clin Nutr 2015; 34: 335 340

[24] Hackl J. Ermittlung des Ernährungsstatus. In: Stein J, Jauch KW, eds. Praxishandbuch klinische Ernährung und Infusionstherapie. Berlin: Springer; 2003: 3-20

[25] Löser C. Unter- und Mangelernährung - Klinik-moderne Therapiestrategien-Budgetrelevanz. Stuttgart: Thieme; 2011

[26] Edington J, Barnes R, Bryan F et al. A prospective randomised controlled trial of nutritional supplementation in malnourished elderly in the community: clinical and health economic outcomes. Clin Nutr 2004; 23: $195-204$

[27] Volkert D. [Practical guideline for nutritional care in geriatric institutions]. Z Gerontol Geriatr 2009; 42: 77 - 87

[28] Kruizenga HM, Seidell JC, de Vet HC et al. Development and validation of a hospital screening tool for malnutrition: the short nutritional assessment questionnaire (SNAQ). Clin Nutr 2005; 24: 75-82

[29] Norman K, Kirchner H, Freudenreich M et al. Three month intervention with protein and energy rich supplements improve muscle function and quality of life in malnourished patients with non-neoplastic gastrointestinal disease - a randomized controlled trial. Clin Nutr 2008; 27: 48- 56

[30] Tucker HN, Miguel SG. Cost containment through nutrition intervention. Nutr Rev 1996; 54: 111-121

[31] Van Cutsem E, Arends J. The causes and consequences of cancerassociated malnutrition. Eur J Oncol Nurs 2005; 9 (Suppl. 02): S51 - 63

[32] Kehlet $\mathrm{H}$. Multimodal approach to control postoperative pathophysiology and rehabilitation. $\mathrm{Br}$ ] Anaesth 1997; 78: 606-617

[33] Bisgaard T, Kehlet $\mathrm{H}$. Early oral feeding after elective abdominal surgery - what are the issues? Nutrition 2002; 18: 944-948

[34] Weimann A, Braga M, Harsanyi L et al. ESPEN Guidelines on Enteral Nutrition: Surgery including organ transplantation. Clinical nutrition (Edinburgh, Scotland) 2006; 25: 224-244 
[35] Braga M, Ljungqvist O, Soeters P et al. ESPEN Guidelines on Parenteral Nutrition: surgery. Clin Nutr 2009; 28: 378 -386

[36] Entlassmanagement [article online]. Available from 2017: https:// www.bundesgesundheitsministerium.de/service/begriffe-von-a-z/e/ entlassmanagement.html 31.12.2018

[37] Bosy-Westphal A, Müller M], Hauner H. Ernährungsberatung: Therapeutischer Nutzen und Förderung der Gesundheit. Dtsch Ärztebl 2015; 112: A1516-A1519

[38] Cederholm T. Reply, Letter to Editor - BMI, FFMI do not seem universally applicable in nutritional assessment \& the place of SGA \& functional evaluation shouldn't be overlooked. Clin Nutr 2015; 35: 237

[39] Keinki C, Seilacher E, Ebel M et al. Information Needs of Cancer Patients and Perception of Impact of the Disease, of Self-Efficacy, and Locus of Control. J Cancer Educ 2016; 31: 610-616
[40] van Veen MR, Beijer S, Adriaans AM et al. Development of a Website Providing Evidence-Based Information About Nutrition and Cancer: Fighting Fiction and Supporting Facts Online. JMIR Res Protoc 2015; 4: e110

[41] Bischoff SC, Arends ], Dörje F et al. S-3 Leitlinie der Deutschen Gesellschaft für Ernährungsmedizin (DGEM) in Zusammenarbeit mit der GESKES und AKE - Künstliche Ernährung im ambulanten Bereich. Aktuel Ernahrungsmed 2013; 38: e101-e154

[42] Hung YC, Bauer J, Horsley P et al. Patient satisfaction with nutrition services amongst cancer patients treated with autologous stem cell transplantation: a comparison of usual and extended care. J Hum Nutr Diet 2013; 27 (Suppl. 02): 333-338 